\title{
Cause of sudden death: giant cell myocarditis
}

\begin{abstract}
Sudden cardiac deaths due to giant cell myocarditis (GCM) are uncommon. Giant cell myocarditis may cause serious heart failure, complete heart block, malignant ventricular arrhythmias. Our case was 34 years old male patient who had sudden loss of consciousness after discussing with another driver in traffic and he died on his way to hospital. The autopsy was performed and in cardiac pathologic examination; chronic inflammatory cells and multinucleated giant cells were observed between myocardial fibers and this finding was found to be consistent with giant cell myocarditis. Giant cell myocarditis is usually seen in young and healthy people. Most of the cases can be diagnosed histologically. The purpose of this study is to aware the forensic experts to evaluate a cardiac case in details and properly.
\end{abstract}

Keywords: sudden cardiac death, giant cell myocarditis, autopsy, arrhythmia
Volume 6 Issue I - 2018

\author{
Kenan Kaya,' Necmi Çekin, ${ }^{1,2}$ Ferhat \\ Yildirim,' Mete Korkut Gülmen,'2 Ahmet \\ Hilal $^{2}$ \\ 'Ministry of Justice, Council of Forensic Medicine, Turkey \\ ${ }^{2}$ Department of Forensic Medicine, Cukurova University School \\ of Medicine, Turkey
}

Correspondence: Kenan Kaya, Ministry of Justice, Council of
Forensic Medicine,Adana, Turkey, Email k_kaya_7I@hotmail.com

Received: November 29, 2017 | Published: January 25, 2018
Abbreviations: GCM, giant cell myocarditis; EMB, endomyocardial biopsy; MRI, magnetic resonance imaging.

\section{Introduction}

Sudden cardiac deaths due to giant cell myocarditis (GCM) are uncommon. Giant cell myocarditis is an inflammatory myocardial disease. It is characterized by inflammation of the heart muscle (myocardium), a condition referred to as myocarditis. Inflammation is caused by widespread infiltration of giant cells associated with other inflammatory cells and cardiac muscles cell destruction. Giant cells are abnormal masses produced by the fusion of inflammatory cells called macrophages. Individuals with giant cell myocarditis may develop abnormal heartbeats, chest pain and, eventually, heart failure. Many individuals eventually require a heart transplant. It mostly occurs in young adults. ${ }^{1}$ It is characterized by resistant sudden arrhythmias and left ventricular failure. Its etiology is still unknown and fatal. GCM rapidly proceeds and clinical view develops very fast in comparison to non-specific lymphocytic myocarditis that usually leads to sudden deaths before any diagnosis. ${ }^{2,3}$ Giant cell myocarditis may cause serious heart failure, complete heart block, malignant ventricular arrhythmias. It usually starts with sudden and severe breathing problem, chest pain, hypotension and orthopnea. Medical treatment is usually unsuccessful. ${ }^{4}$ GCM cases are very rare and a sometimes create serious diagnostic problems of the sudden cardiac deaths if not properly investigated and evaluated.

\section{Case presentation}

Our case was 34years old male patient who had sudden loss of consciousness after discussing with another driver in traffic and he died on his way to hospital. The case brought to the Morgue Department of The Institute of Forensic Medicine of Adana, Turkey. Autopsy was performed and evaluated by two forensic medicine specialist and consulted to a pathologist. On internal examination, the heart weighed $340 \mathrm{~g}$ and thickness of left ventricular wall was $1,3 \mathrm{~cm}$, that of right ventricle was $0,1-0,3 \mathrm{~cm}$. Papillary muscles in both ventricles were fibrotic and showed disarrangement. Myocardial sections showed areas of fibrosis and dark-mottled areas. Microscopic examination of myocardial sections revealed connective tissue replacing normal myocardial muscle bundles; diffuse lymphohistiositic inflammatory cell infiltration, multinuclear giant cells (Figures 1). Microscopic sections of other internal organs showed no pathology other than hyperemia. On the basis of these findings, the final diagnosis was giant-cell myocarditis.
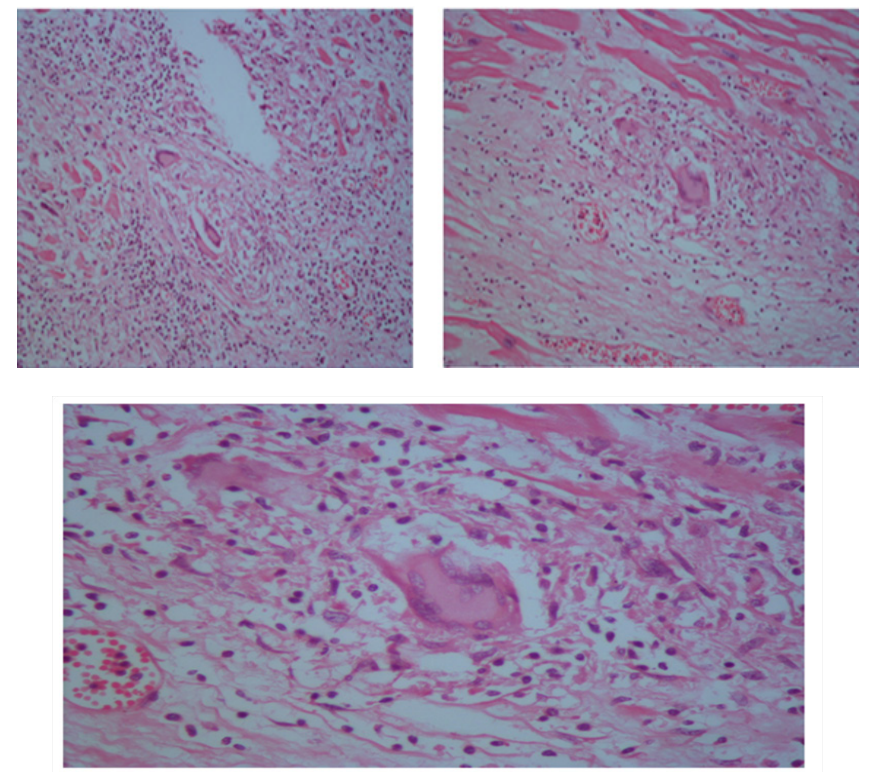

Figures I Myocardial lymphohistiositic inflammatory cell infiltration, multinuclear giant cells (HEX 100, HEX 200, HEX 400).

\section{Discussion}

Giant cell myocarditis is usually seen in young and healthy people. It is thought to be a form of autoimmune myocarditis. That autoimmune origin is supported by the association of giant cell myocarditis with autoimmune diseases and the presence of giant cells in posttransplanted cardiac tissues. The origin of the giant cells is still under investigations. Furthermore, there were few cases of GCM following 
immunotherapy that were reported in the literature, emphasizing on its auto-immune etiology. ${ }^{5,6}$ Most of the cases can be diagnosed histologically. Endomyocardial biopsy (EMB) is a highly-specialized procedure that is associated with some controversy with the advances in echocardiography and cardiac magnetic resonance imaging (MRI), the large majority of cardiac diseases can be diagnosed by these noninvasively procedures, thus the under-sampling and biopsy area is limited to the right side of the intraventricular septum. In spite of all these limitations, there still remains a group of pathological conditions that require biopsy for a conclusive diagnosis such as idiopathic myocarditis, amyloidosis, sarcoidosis and giant cell myocarditis. ${ }^{7}$

GCM is an idiopathic myocarditis, with large necrotic areas in addition to numerous multinucleated giant cells (myocytes or macrophage origin) as well as lymphocytes, macrophages, plasma cells and eosinophils. ${ }^{8}$ It is associated with poor prognosis. Giant cell myocarditis case is presented as a very rare entity in literature whose diagnosis could only be made with microscopic examination of the heart and it should be in differential diagnosis of sudden death cases. Forensic experts should always be aware to evaluate a cardiac case in details and properly, and when myocarditis is suspected clinically blood tests including detailed history, ECG and cardiac enzymes, serum anticard antibody presence should be taken; imaging methods including echocardiography and cardiac MR imaging should be performed. Patients presenting with myocarditis should undergo cardiac MRI to better define cardiac anatomy. Cardiac biopsy can be helpful in making the correct diagnosis and ensuring that correct timely treatment is administered. ${ }^{7,9}$ Giant cell myocarditis can be confused with cardiac sarcoidosis. Therefore all suspected cases should be reviewed by an experienced cardiac pathologist. Giant cell myocarditis and cardiac sarcoidosis are two disorders that only recently have been considered as distinct histopathological entities. Both are potentially lethal myocarditis forms that are infectiousnegative and immune-mediated. For both of them the dismal prognosis can be improved by early diagnosis and tailored immunosuppressive therapy. Although both entities are perceived as rare conditions, two recent studies have suggested the possibility that they may be much more common than previously suspected, for instance in the group of young patients with apparently idiopathic atrio-ventricular blocks or in patients with atrial fibrillation. As for other diseases, the most severe cases are first clinically recognized, but then the medical community realizes that such cases represent only the tip of the iceberg and that less severe forms are much more prevalent and under diagnosed. In the case of GCM there is no distinctive clinical presentation, but, as for other forms of myocarditis, GCM can present as acute or chronic heart failure, arrhythmia, myocardial necrosis with normal coronary arteries, or a life-threatening condition (cardiac arrest or carcinogenic shock). Some clinical hints are the severity of cardiac symptoms, the lack of response to standard cardiological care and the association in the patient or in family members with other extra-cardiac autoimmune conditions, such as autoimmune thyroid disease, ulcerative colitis, etc. ${ }^{10-12}$ It is reported that GCM has a relationship with an auto immune diseases such as Sjogren's syndrome, giant cell arteritis, thymoma, myasthenia gravis, chronic active hepatitis, Hashiomoto's thyroiditis, ulcerative colitis and Crohn's disease. , $^{2,10,11}$

\section{Conclusion}

A very rare case of giant cell myocarditis should be included in the differential diagnosis of sudden suspicious deaths and especially cardiac deaths. The diagnosis was made possible only by microscopic examination of the heart.

\section{Acknowledgements}

None.

\section{Conflict of interest}

The author declares that there is no conflict of interest.

\section{References}

1. Sheppard M, Davies MJ. Cardiac hypertrophy, myocarditis and cardiomyopathy in Practical Cardiovascular Pathology. 1st ed. USA: Oxford University Press; 1998. p. 103-148.

2. Shariff S, Straatman L, Allard M, et al. Novel associations of giant cell myocarditis: two case reports and a review of the literature. Can J Cardiol. 2004;20(5):557-561.

3. Karapur PG, Dhaded AV, Udayakumar M. Idiopathic giant cell myocarditis-a case report and review of literature. Indian J Pathol Microbiol. 2003;46(2):241-243.

4. Demir M, Tasal A, San M. Miyokardit. Turkey Clinical J Med Sci. 2006;26:441-448.

5. Bracamonte Baran W, Čiháková D. Cardiac Autoimmunity: Myocarditis. Adv Exp Med Biol. 2017;1003:187-221.

6. Reuben A, Petaccia de Macedo M, McQuade J, et al. Comparative immunologic characterization of autoimmune giant cell myocarditis with ipilimumab. OncoImmunology. 2017;6(12):e1361097.

7. Khan T, Selvakumar D, Trivedi S, et al. The value of endomyocardial biopsy in diagnosis and guiding therapy. Pathology. 2017;49(7):750-756.

8. Frederick J Schoen. The heart, Robbins and Cotran Pathological Basis of Disease. 7th ed. Philadelphia, USA; 2005. p. 608-609.

9. Laissy JP, Hyafil F, Feldman LJ, et al. Differentiating acute myocardial infarction from myocarditis: diagnostic value of early-and delayedperfusion cardiac MR imaging. Radiology. 2005;237(1):75-82.

10. Lassner D, Kuhl U, Siegismund CS, et al. Improved diagnosis of idiopathic giant cell myocarditis and cardiac sarcoidosis by myocardial gene expression profiling. Eur Heart J. 2014;35(32):2186-2195.

11. Blawet LA, Cooper LT. Idiopathic giant cell myocarditis and cardiac sarcoidosis. Heart Fail Rev. 2013;18(6):733-746.

12. Okura Y, Dec GW, Hare JM, et al. A clinical and histopathologic comparison of cardiac sarcoidosis and idiopathic giant cell myocarditis. Am J Coll Cardiol. 2003;41(2):322-329. 